\title{
Charged Higgs production with a top in MC@NLO
}

\author{
Tilman Plehn* \\ Institut für Theoretische Physik, \\ Universität Heidelberg, Germany \\ E-mail: plehn@uni-heidelberg.de
}

\section{Carole Weydert}

Laboratoire de Physique Subatomique et de Cosmologie, UJF,

CNRS/IN2P3,

Grenoble, France

E-mail: weydertelpsc.in2p3.fr

\begin{abstract}
The production in association with a top quark is the most promising search channel for charged Higgs bosons at the LHC. We review its theoretical description including next-to-leading order corrections and the combination with a parton shower. The latter allows us to for the first time answer questions about the kinematics of all jets in the process. We then describe the consistent subtraction of intermediate states and present new results about the bottom mass uncertainty impacting the parton densisities.
\end{abstract}

Prospects for Charged Higgs Discovery at Colliders

27-30 September 2010

Uppsala University, Sweden

\footnotetext{
*Speaker.
} 


\section{Four and five flavors}

As has been known for a long time [1,2] processes relying on gluon splitting into an initial state $b \bar{b}$ pair can be described by generating a universal bottom content in the proton via the DGLAP evolution and including it as a parton density. In our case this means that to leading order we compute the process $p_{5} p_{5} \rightarrow t H^{-}$with five quark flavors inside the proton $[3,4,5,6]$. Following Figure 1 we can as well limit the quark content of the proton to four light flavors and only generate initial state bottom quarks via gluon splitting, so our leading order process is $p_{4} p_{4} \rightarrow \bar{b} t H^{-}$[7]. The index of the proton indicates that this implies different proton pictures, i.e. different parton densities and $\alpha_{s}$ values.

To all orders in perturbation theory the two approaches are equivalent. The difference between them is the ordering in perturbative QCD. The DGLAP equation resums collinear logarithms which appear at every fixed order in $\alpha_{s}$. Since they diverge for massless quarks there is no question that for those the logarithms have to be resummed, to obtain finite and stable rate predictions. For bottoms the logarithms have the form $\log \left(M / m_{b}\right)$ with the process dependent hard scale $M \sim m_{t}+m_{H}$. They are regularized and reside in the perturbative regime $m_{b} \gg \Lambda_{\mathrm{QCD}}$.

Because of these logarithms, comparisons at leading order have to be taken with a grain of salt. The renormalization and factorization scale dependences in both processes are large and the predicted rates correspondingly uncertain. In the five-flavor scheme the factorization scale dependence of the total rate is approximately logarithmic and has a one-to-one correspondence to the collinear logarithm [1]. Setting the factorization scale to $\mu_{F} \equiv M$ implicitly assumes the existence of large logarithms all the way to $\log \left(M / m_{b}\right)$. A careful study of the kinematics of gluon splitting generating the logarithms shows that bottom densities combined with an incoming gluon or bottom never generate as large logarithms, due to the steep drop of the gluon parton densities towards large $x[2,4,8]$. Only a consistent (lower) choice of the bottom factorization scale ensures a stable perturbative behavior of the five-flavor production rate. For $t H^{-}$production these effects are not huge, while they completely change the balance of the different production modes for example in the case of charged Higgs pair production [9]. Moreover, given that we have fully understood NLO results for the five-flavor [4, 5, 6] and four-flavor [7] descriptions of $t H^{-}$production we do not expect a huge discrepancy between the different rate predictions.

Expanding the argument to distributions there appear slight differences between the two calculations. The distributions of the heavy top and Higgs are computed with the same perturbative accuracy as the respective total rates. Luckily, we know for example from the production of supersymmetric particles [10] that the distributions of heavy particles produced in pairs are remarkably stable with respect to higher orders in QCD, so again we do not expect large discrepancies.
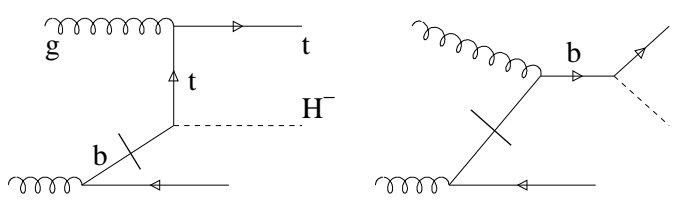

Figure 1: Feynman diagrams for associated top-Higgs production at the LHC. 


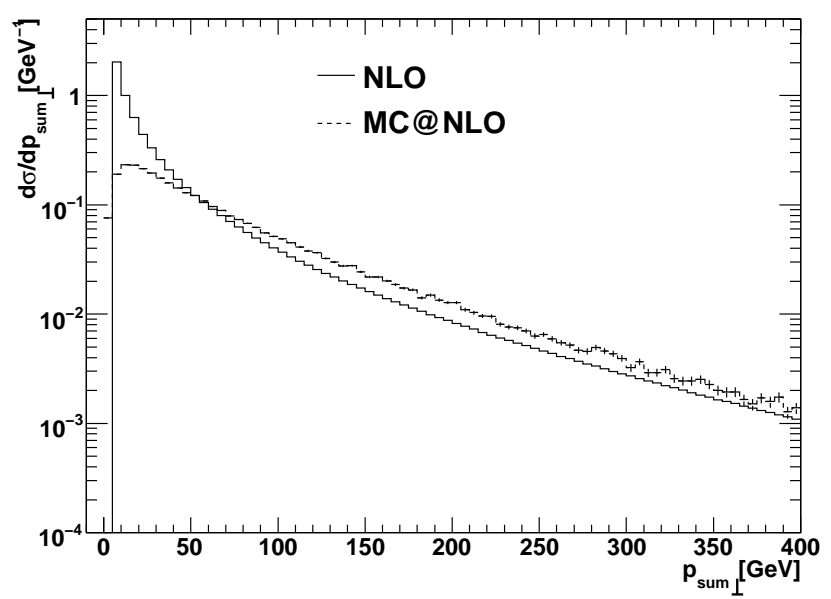

Figure 2: Transverse momentum distribution of the top-Higgs pair, simulated at fixed order (NLO, five flavors) and including the parton shower [6]. The assumed charged Higgs mass is $300 \mathrm{GeV}$.

\section{MC@NLO and jet radiation}

The main difference between the NLO four-flavor and five-flavor calculations appears in the bottom jet distributions. On the one hand, the motivation for using bottom partons is that in most events we will not see the forward and comparably soft bottom jet, and integrating over its phase space produces the logarithmic rate enhancement. On the other hand, modern analysis methods are able to make use of such features which are only visible in a fraction of the events, so our simulation has to describe them correctly.

To leading order, in the five-flavor scheme the heavy pair recoils against a strictly longitudinal bottom. Only at NLO this recoiling jet acquires a finite $p_{T, b}[5]$. In the four-flavor scheme the recoiling bottom is realistically described already at leading order. At first glance this looks like a case for a four-flavor calculation, at least when looking at distributions. However, from the moderate size of the collinear logarithm we know that $p_{T, b} \ll M$, i.e. in the bulk of its phase space the bottom jet is correctly described by a parton shower and not by a hard matrix element. What we should really do is combine either the four-flavor or the five-flavor description with a parton shower. This is precisely what MC@NLO does. The impact of the parton shower becomes obvious in the $p_{T}$ distribution of the top-Higgs system shown in Figure 2. At fixed order it diverges while the resummed collinear logarithms in the parton shower correctly describe the low- $p_{T}$ regime.

Combining the parton shower with the matrix element to NLO ensures that we correctly describe all jet radiation while the total rate and the hard distributions are at NLO accuracy. This way we do not need an external normalization, as it used to be the case for PYTHIA and HERWIG. With a built-in NLO rate prediction we can answer questions of the kind [6]:

- What are the transverse momentum or rapidity spectra of the radiated $b$ jet?

- Are these $b$ spectra the same as for light-flavor initial state radiation?

- Is the $b$ jet from the top decay always harder than the $b$ jet from initial state radiation?

- How likely is it that we observe or tag this radiated $b$ jet?

- How often do we see light-flavor or $b$ jets given one $b$ jet from the top decay has been observed? 


\begin{tabular}{cc||c|c|c|c|c||c|c|c|c|c|}
\multicolumn{1}{l}{} & \multicolumn{6}{c||}{} & \multicolumn{6}{c||}{$m_{H}=300 \mathrm{GeV}$} & \multicolumn{5}{c|}{$m_{H}=800 \mathrm{GeV}$} \\
\hline & $p_{T}^{\min } \backslash \eta^{\max }$ & 2.5 & 2.0 & 1.5 & 1.0 & 0.5 & 2.5 & 2.0 & 1.5 & 1.0 & 0.5 \\
\hline$\left(b\right.$ jet, $\left.t_{\ell}\right)$ & $25 \mathrm{GeV}$ & 17.8 & 14.3 & 10.0 & 5.7 & 2.3 & 21.4 & 17.1 & 12.1 & 7.1 & 3.0 \\
& $45 \mathrm{GeV}$ & 12.9 & 10.6 & 7.6 & 4.5 & 1.8 & 16.6 & 13.7 & 9.9 & 6.0 & 2.5 \\
& $65 \mathrm{GeV}$ & 9.4 & 8.0 & 5.9 & 3.5 & 1.6 & 12.3 & 10.5 & 7.9 & 4.9 & 2.0 \\
& $85 \mathrm{GeV}$ & 7.2 & 6.4 & 4.8 & 3.0 & 1.4 & 9.7 & 8.5 & 6.5 & 4.1 & 1.7 \\
\hline (light jet, $\left.t_{\ell}\right)$ & $25 \mathrm{GeV}$ & 45.9 & 40.0 & 32.7 & 23.9 & 13.0 & 54.8 & 48.8 & 41.0 & 31.0 & 17.9 \\
& $45 \mathrm{GeV}$ & 32.4 & 27.8 & 22.3 & 16.1 & 9.0 & 41.7 & 36.7 & 30.5 & 23.0 & 13.7 \\
& $65 \mathrm{GeV}$ & 22.3 & 18.8 & 14.7 & 10.4 & 5.8 & 30.9 & 27.0 & 22.2 & 16.5 & 10.2 \\
& $85 \mathrm{GeV}$ & 16.2 & 13.4 & 10.3 & 7.3 & 4.2 & 23.6 & 20.5 & 16.6 & 12.1 & 7.4 \\
\hline (light jet, $\left.t_{h}\right)$ & $25 \mathrm{GeV}$ & 94.9 & 91.0 & 84.3 & 72.2 & 48.4 & 95.8 & 92.5 & 86.3 & 75.0 & 52.0 \\
& $45 \mathrm{GeV}$ & 83.2 & 79.2 & 72.3 & 61.0 & 39.9 & 87.1 & 83.3 & 76.8 & 65.7 & 45.2 \\
& $65 \mathrm{GeV}$ & 60.9 & 57.3 & 51.7 & 43.2 & 28.8 & 70.5 & 66.9 & 61.3 & 51.9 & 35.9 \\
& $85 \mathrm{GeV}$ & 44.4 & 41.5 & 37.1 & 31.1 & 21.3 & 56.2 & 53.3 & 48.6 & 41.0 & 28.7
\end{tabular}

Table 1: Probability (\%) to see a $b$ or light jet in a given detector region, after one $b$ jet, most likely from the top decay, is observed. The top decays leptonically or hadronically while the charged Higgs is stable [6].

As an example, we answer the last question in Table 1 [6]. The starting point, motivated by the usual analyses, is that one bottom jet from the top decay should always be observed. The additional bottom jet arises from initial state gluon splitting. In the five-flavor scheme to leading order the probability of seeing such a bottom jet within the fiducial volume of the detector is zero. Including the NLO corrections with a reliable $p_{T, b}$ spectrum over the entire range indicates that in roughly $18 \%$ of the events we can tag the radiated bottom jet given $p_{T, b}>25 \mathrm{GeV}$ and $\left|\eta_{b}\right|<2.5$ for $m_{H}=300 \mathrm{GeV}$. Combined with typical tagging efficiencies this translates into roughly $10 \%$ of the inclusive $t H^{-}$events with a $b$ tag. Because of the collinear enhancement the radiated bottom jet becomes harder for larger Higgs masses, but the generic picture of roughly $10 \%$ of all events including a tagged forward bottom does not change.

In addition, we can ask if the appearance of a forward jet can be viewed as a sign for initialstate gluon splitting into two bottoms. Table 1 indicates that for a leptonic top decay, i.e. no additional hard jets in the event, it is two to three times as likely to observe a light-flavor jet from initial state or final state radiation than a bottom jet. When we in addition allow for jets from a hadronic top decay almost all events will see an additional jet with $p_{T, j}>25 \mathrm{GeV}$ and $\left|\eta_{j}\right|<2.5$. This percentile only drops once $p_{T, j}$ reaches the Jacobian peak of the top decay.

Again, only the combination of a hard NLO matrix element with the parton shower allows us to systematically compare such kinematic features of bottom jet radiation, light-flavor jet radiation, and top decay jets, as shown in Table. 1 [6].

\section{Bottom mass and bottom partons}

Bottom parton densities are based on the splitting of an off-shell gluon into a pair of massive bottoms. While for light-flavor quarks the splitting threshold is of the order of $\Lambda_{\mathrm{QCD}}$ and hence not numerically relevant, for bottoms it is in the range of perturbative QCD. This makes it a relevant input parameter in the computation of bottom parton densities, which has to be tested [11]. To first approximation, a shift in the bottom mass changes the logarithmic parton densities by 


\begin{tabular}{c||l|l|l|l||l|l||l|l}
\multicolumn{1}{l||}{} & \multicolumn{4}{c||}{$m_{H}=200 \mathrm{GeV}$} & \multicolumn{2}{c||}{$m_{H}=500 \mathrm{GeV}$} & \multicolumn{2}{c}{$m_{H}=800 \mathrm{GeV}$} \\
\hline \multicolumn{1}{l||}{} & \multicolumn{2}{c|}{$7 \mathrm{TeV}$} & \multicolumn{2}{c|}{$14 \mathrm{TeV}$} & \multicolumn{2}{c}{$14 \mathrm{TeV}$} & \multicolumn{2}{c}{$14 \mathrm{TeV}$} \\
\hline$m_{b}$ & $\sigma[\mathrm{pb}]$ & $\sigma / \sigma_{4.75}$ & $\sigma[\mathrm{pb}]$ & $\sigma / \sigma_{4.75}$ & $\sigma[\mathrm{pb}]$ & $\sigma / \sigma_{4.75}$ & $\sigma[\mathrm{pb}]$ & $\sigma / \sigma_{4.75}$ \\
\hline 4.25 & 0.1845 & 1.055 & 1.279 & 1.049 & 0.1168 & 1.045 & 0.01989 & 1.044 \\
4.50 & 0.1796 & 1.026 & 1.248 & 1.025 & 0.1142 & 1.021 & 0.01945 & 1.021 \\
4.75 & 0.1750 & 1.0 & 1.219 & 1.0 & 0.1118 & 1.0 & 0.01905 & 1.0 \\
5.00 & 0.1708 & 0.976 & 1.192 & 0.978 & 0.1096 & 0.980 & 0.01868 & 0.981 \\
5.25 & 0.1668 & 0.953 & 1.166 & 0.957 & 0.1074 & 0.961 & 0.01832 & 0.977
\end{tabular}

Table 2: Absolute and relative production rates for $t H^{-}$production at NLO, varying the input bottom mass in the on-shell scheme. The coupling is fixed by $\tan \beta=30$, and the renormalization scale is $\mu=\left(m_{t}+m_{H}\right) / 2$.

$$
\log \frac{M}{m_{b}} \rightarrow \log \frac{M}{m_{b}+\delta m_{b}}=\log \frac{M}{m_{b}}-\log \left(1+\frac{\delta m_{b}}{m_{b}}\right) \simeq \log \frac{M}{m_{b}}-\frac{\delta m_{b}}{m_{b}} .
$$

For well motivated applications of the bottom parton densities $\left(M \gg m_{b}\right)$ the uncertainly due to the bottom mass becomes increasingly irrelevant. For example, a logarithm of the order log $100 \sim 4.6$ reduces the relative error from the bottom mass input by this factor 1/4.6.

Because in the matrix element squared of the hard process the bottom mass only enters power suppressed we set it to zero in that part of the calculation. This includes the collinear divergence which we regularize in dimensional regularization and move into the parton densities. It is disputable if together with the input bottom mass, i.e. the threshold of the gluon splitting, we should also vary the bottom Yukawa coupling. However, for large enough $\tan \beta$ the change of the production rate with the bottom Yukawa coupling is trivially given by $\sigma \propto y_{b}^{2}$. If we evaluate the bottom Yukawa in the $\overline{\mathrm{MS}}$ scheme, changing the on-shell bottom mass by $\pm 0.5 \mathrm{GeV}$ changes $y_{b}(M)$ by roughly $\pm 13 \%$ and the Higgs cross section by $\pm 25 \%{ }^{1}$

In comparison, varying the bottom mass in the parton densities over $4.25 \cdots 5.25 \mathrm{GeV}$ for $7 \mathrm{TeV}$ collider energy leads to a $\pm 5 \%$ window for a Higgs mass of $200 \mathrm{GeV}$, as shown in Table 2. The change in rate is half of the change in the bottom mass, roughly in line with Eq.(3.1). For larger collider energies and for larger Higgs masses the relative change in rate slightly decreases, again as expected. This again confirms Eq.(3.1) and its conclusion that the effect of the bottom mass through the parton densities is strongly suppressed in comparison with the shift in the bottom Yukawa.

\section{Small Higgs masses}

For small Higgs masses a problem arises in the NLO corrections to the process $b g \rightarrow t H^{-}$: for example the $\mathscr{O}\left(y_{t}^{2} \alpha_{s}^{2}\right)$ subprocess

$$
g g / q \bar{q} \rightarrow t H^{-} \bar{b}
$$

includes diagrams with on-shell $g g \rightarrow t \bar{t}$ production and a subsequent decay $\bar{t} \rightarrow H^{-} \bar{b}$. They only include a two-particle production phase space, $\sigma_{t \bar{t}} \times \mathrm{BR}_{\bar{t} \rightarrow H^{-} \bar{b}}$, and therefore numerically dominate.

\footnotetext{
${ }^{1}$ Note that in the MC@NLO implementation the starting value $m_{b}\left(m_{b}\right)=4.23 \mathrm{GeV}$ is fixed, corresponding to an on-shell value of $4.87 \mathrm{GeV}$. Varying the input bottom mass will not actually change the bottom Yukawa coupling.
} 

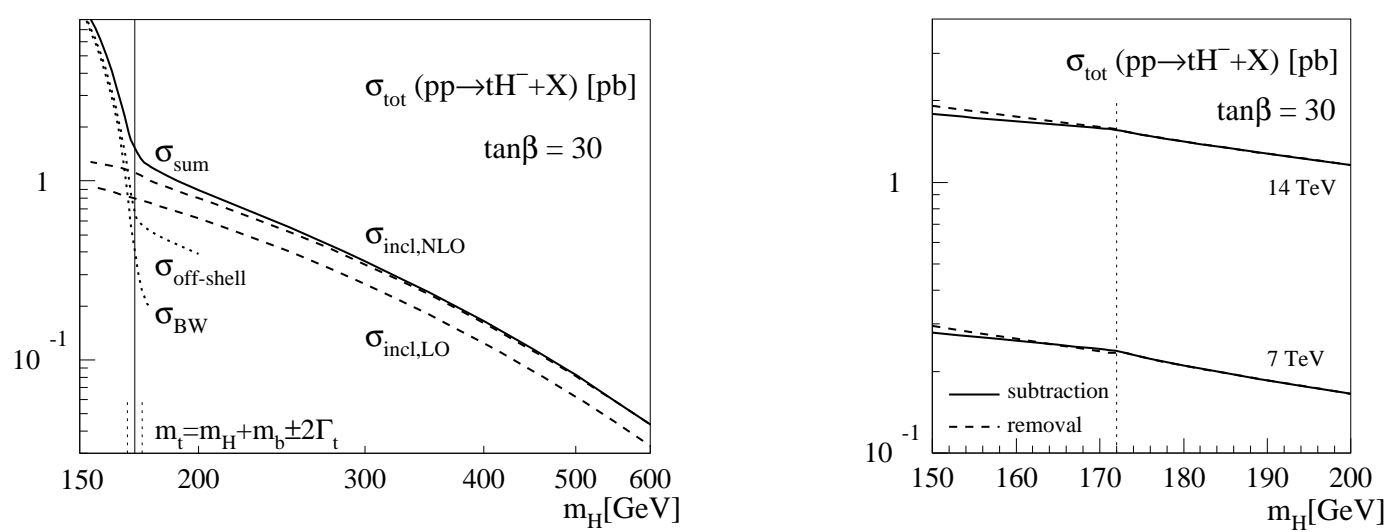

Figure 3: Total cross sections for top-Higgs production at the LHC including small Higgs masses. Left: PROSPINO implementation [5]; Right: MC@ NLO implementation [6], for the correct diagram subtraction and the inconsistent diagram removal.

From a field theory perspective there exists no problem with these on-shell diagrams, aside from the usual complications of intermediate on-shell particles. To compute LHC rates and distributions we can introduce a width $\Gamma_{t}$ or apply more advances schemes in analogy to four fermion production at LEP [12]. From Tevatron top analyses we expect that the introduction of a width while keeping the spin correlation of the top quark will be completely sufficient.

The two-fold reason for separating top pairs from top-Higgs production is phenomenological: first, the two processes have different kinematic features and therefore require different analyses based on different (simulated) event samples. Second, the QCD corrections to the two processes should not be expected to be identical. Therefore, we first simulate top pair production including QCD effects and normalized to the measured rate. Top-Higgs production then corresponds to an event sample which we can add to the top pair sample without any double counting.

The appropriate treatment of such intermediate states in different $2 \rightarrow 2$ production channels has been developed in PROSPINO [10] and the NLO $p p \rightarrow H^{-} t$ calculation [5]. It was then independently applied to single top production in MC@ NLO as so-called diagram subtraction [13]. The challenge is to define local subtraction terms which remove the on-shell process $p p \rightarrow t \bar{t}$ from the real emission contribution Eq.(4.1). Interference between the two processes of course counts towards new physics, i.e. top-Higgs production. This is why diagram removal schemes on the amplitude level are not useful.

In terms of the momentum squared $s_{b h}$ flowing through the intermediate top propagator and the corresponding residue $|\mathscr{M}|^{2}\left(s_{b h}\right)$ we add a subtraction term to the otherwise unmodified BreitWigner top propagator

$$
\frac{|\mathscr{M}|^{2}\left(s_{b h}\right)}{\left(s_{b h}-m_{t}^{2}\right)^{2}+m_{t}^{2} \Gamma_{t}^{2}}-\frac{|\mathscr{M}|^{2}\left(m_{t}^{2}\right)}{\left(s_{b h}-m_{t}^{2}\right)^{2}+m_{t}^{2} \Gamma_{t}^{2}} \Theta\left(\hat{s}-4 m_{t}^{2}\right) \Theta\left(m_{t}-m_{H}\right),
$$

including the appropriate constrained phase space [10]. This expression holds independently of the value of the regulator $\Gamma_{t}$. In the PROSPINO implementation with quasi-stable supersymmetric particles we use the gauge invariant narrow width limit to uniquely define the subtraction term

$$
\lim _{\Gamma_{t} \ll m_{t}} \frac{m_{t} \Gamma_{t}}{\left(s_{b h}-m_{t}^{2}\right)^{2}+m_{t}^{2} \Gamma_{t}^{2}}=\pi \delta\left(s_{b h}-m_{t}^{2}\right) .
$$




\begin{tabular}{c||c|c|c||c|c|c|}
\multicolumn{1}{l||}{} & \multicolumn{3}{c||}{$\sigma_{\mathrm{NLO}}(7 \mathrm{TeV})$} & \multicolumn{3}{c|}{$\sigma_{\mathrm{NLO}}(14 \mathrm{TeV})$} \\
\hline$m_{H}$ & $\mu=\mu_{0}$ & $\mu=\mu_{0} / 3$ & $\mu=3 \mu_{0}$ & $\mu=\mu_{0}$ & $\mu=\mu_{0} / 3$ & $\mu=3 \mu_{0}$ \\
\hline 200 & 0.1777 & 0.1944 & 0.1605 & 1.237 & 1.398 & 1.133 \\
250 & 0.1036 & 0.1101 & 0.0934 & 0.7911 & 0.8752 & 0.7232 \\
300 & 0.06266 & 0.06562 & 0.05618 & 0.5212 & 0.5707 & 0.4743 \\
350 & 0.03887 & 0.04027 & 0.03467 & 0.3516 & 0.3813 & 0.3185 \\
400 & 0.02467 & 0.02533 & 0.02189 & 0.2421 & 0.2607 & 0.2184 \\
450 & 0.01598 & 0.01627 & 0.01410 & 0.1698 & 0.1818 & 0.1527 \\
500 & 0.01053 & 0.01065 & 0.00925 & 0.1211 & 0.1290 & 0.1085
\end{tabular}

Table 3: NLO Production rates in $\mathrm{pb}$ for $p p \rightarrow t H^{-}$production in the five-flavor scheme, using the input parameters defined by the LHC Higgs cross sections working group. The central renormalization and factorization scales are chosen as $\mu_{0}=\left(m_{t}+m_{H}\right) / 4[4,5]$. The bottom Yukawa scales with $\tan \beta=30$.

In contrast, the MC@NLO implementation includes top decays, so we identify the regulator with the physical anomalous top width.

In Figure 3 we show the different contributions to associated top-Higgs production at the LHC [5]. Top pair production including a Breit-Wigner propagator and the appropriate branching ratio is labelled $\sigma_{\mathrm{BW}}$. It dominates for small Higgs masses and quickly becomes numerically irrelevant in the threshold region. This description might be improved by including all off-shell diagrams contributing to $t H^{-} \bar{b}$ production. However, $\sigma_{\text {off-shell }}$ only shows an effect above threshold, where it corresponds to a subset of the NLO top-Higgs diagrams. Instead, we use the complete subtracted $\sigma_{\text {incl,NLO }}$ which together with the on-shell top pair production gives the full rate $\sigma_{\text {sum. }}$. The resulting $K$ factor for the subtracted $t H^{-}$production is flat across the threshold $m_{H}=m_{t}$.

As a side remark, the four-flavor prediction for $p \rightarrow \bar{b} t H^{-}$[7] includes the on-shell top contribution to leading order, regularized by the physical top width. However, the separation into two distinctly different processes would work the same way, for example if one would want to normalize the top-pair production rate to data.

\section{Outlook}

We have reviewed the status of theoretical predictions for the production rate of associated $t H^{-}$production in a two-Higgs doublet model. Two schemes describe bottom initiated processes at hadron colliders, with or without bottoms densities inside the proton. To all orders in perturbative QCD they are equivalent, but to leading order they give different results. Both schemes have been implemented to NLO, and we find reasonable agreement for the production rates.

For distributions the difference between these two schemes is not the main issue. The relevant collinear bottom and light-flavor jet radiation is be poorly described by either of them and requires a combination with a parton shower. MC@NLO describes the kinematics of bottom and light-flavor jet radiation reliably over the entire phase space and allows us to study the features of radiated bottom jets, radiated light-flavor jets, and jets from the top decay.

One yet unstudied issue in the five-flavor scheme is the dependence of the rate on the bottom mass assumed for the bottom parton densities. The effect of a shift in the input bottom mass is suppressed by the same collinear logarithm which justifies the use of bottom parton densities. Its numerical impact does not exceed a few per-cent. 
For light charged Higgs bosons it is crucial that we consistently combine higher order corrections of the kind $p p \rightarrow t H^{-} \bar{b}$ with top pair production followed by an anomalous decay $\bar{t} \rightarrow H^{-} \bar{b}$. There exists a unique prescription how to separate the two processes using a point-wise phase space subtraction. We have reviewed the basic features of this PROSPINO subtraction scheme and its MC@NLO implementation.

Finally, we give the NLO cross section predictions for the five-flavor scheme using the input parameters defined by the LHC Higgs cross section working group. For two different LHC collider energies and a range of renormalization and factorization scales they are shown in Table 3.

We would like to thank the organizers of this workshop series for the very inspiring and constructive atmosphere. Moreover, we would like to thank Michael Krämer for many constructive discussions.

\section{References}

[1] J. C. Collins and W. K. Tung, Nucl. Phys. B 278, 934 (1986); M. A. Aivazis, J. C. Collins, F. I. Olness and W. K. Tung, Phys. Rev. D 50, 3102 (1994); F. I. Olness and W. K. Tung, Nucl. Phys. B 308, 813 (1988); M. Krämer, F. I. Olness and D. E. Soper, Phys. Rev. D 62, 096007 (2000); J. Alwall and J. Rathsman, JHEP 0412, 050 (2004).

[2] E. Boos and T. Plehn, Phys. Rev. D 69, 094005 (2004).

[3] R. M. Barnett, H. E. Haber and D. E. Soper, Nucl. Phys. B 306, 697 (1988); D. A. Dicus, S. Willenbrock, Phys. Rev. D39, 751 (1989); A. C. Bawa, C. S. Kim and A. D. Martin, Z. Phys. C 47, 75 (1990); V. D. Barger, R. J. Phillips and D. P. Roy, Phys. Lett. B 324, 236 (1994).

[4] T. Plehn, Phys. Rev. D 67, 014018 (2003); S. H. Zhu, Phys. Rev. D 67, 075006 (2003).

[5] E. L. Berger, T. Han, J. Jiang et al., Phys. Rev. D71, 115012 (2005).

[6] C. Weydert, S. Frixione, M. Herquet et al., Eur. Phys. J. C67, 617-636 (2010).

[7] S. Dittmaier, M. Krämer, M. Spira et al., [arXiv:0906.2648 [hep-ph]].

[8] J. M. Campbell, R. K. Ellis, F. Maltoni et al., Phys. Rev. D67, 095002 (2003); F. Maltoni, Z. Sullivan and S. Willenbrock, Phys. Rev. D 67, 093005 (2003).

[9] A. Krause, T. Plehn, M. Spira and P. M. Zerwas, Nucl. Phys. B 519, 85 (1998); A. A. Barrientos Bendezu and B. A. Kniehl, Nucl. Phys. B 568, 305 (2000); O. Brein and W. Hollik, Eur. Phys. J. C 13, 175 (2000); S. Moretti and J. Rathsman, Eur. Phys. J. C 33, 41 (2004); A. Alves and T. Plehn, Phys. Rev. D71, 115014 (2005).

[10] W. Beenakker, R. Höpker, M. Spira and P. M. Zerwas, Nucl. Phys. B 492, 51 (1997); W. Beenakker, M. Klasen, M. Krämer, T. Plehn, M. Spira, and P. M. Zerwas, Phys. Rev. Lett. 83, 3780 (1999); www.thphys.uni-heidelberg.de/ plehn/prospino

[11] A. D. Martin, W. J. Stirling, R. S. Thorne et al., Eur. Phys. J. C70, 51-72 (2010).

[12] see e.g. A. Denner, S. Dittmaier, M. Roth et al., Nucl. Phys. B724, 247-294 (2005).

[13] S. Frixione, E. Laenen, P. Motylinski et al., JHEP 0807, 029 (2008). 